Bol. Acad. peru. leng. 53. 2012 (29-41)

\title{
EL REANÁLISIS EN LA DIACRONÍA CASTELLANA
}

\author{
LE RÉANALYSE DANS LA DIACHRONIE CASTILLANE
}

\section{THE REANALYSIS IN THE SPANISH DIACHRONY}

\author{
Raymundo Casas Navarro \\ Universidad Nacional Mayor de San Marcos
}

\section{Resumen:}

En este artículo, trabajamos con la categoría de reanálisis para echar luces sobre una serie de cambios ocurridos en la historia de la lengua castellana, y, de ese modo, superamos el nivel de la mera descripción para alcanzar el objetivo medular de la ciencia: la explicación teórica. Con el fin de lograr nuestro cometido, nos sustentamos en el framework elaborado por Langacker (1977). El marco teórico langackeriano establece un concepto nítido de reanálisis y elabora una pulcra distinción de sus tipos, causas y efectos. En primer término, haremos una escueta presentación de las nociones de Langacker, sobre todo, en lo que respecta a los tipos de reanálisis. Luego, nos centraremos en algunos procesos diacrónicos castellanos que se pueden dilucidar en términos de la categoría langackeriana. Finalmente, expondremos algunos corolarios que se derivan de este framework para apuntalar una visión cognitiva sobre el cambio lingüístico. 


\section{Résumé:}

Dans cet article, on travaille avec la catégorie de reanályse pour éclaircir une série de changements arrivés dans l'histoire de la langue castillane, et de cette façon on remonte le niveau d'une simple description pour atteindre l'objectif médullaire de la science: l'explication théorique. Dans ce but on utilise le framework réalisé par Langacker (1977) pour renforcer notre recherche. Le cadre théorique langackerien établit un concept transparent de reanályse et élabore une distinction soignée de ses types, causes et effets. En premier terme, Nous ferons une présentation sobre des notions de Langacker, surtout, en ce qui concerne aux types de reanályse. Après, on se centrera en quelques procès diachroniques castillans qui puissent élucider en termes de la catégorie langackerienne. Finalement, On exposera quelques corolaires qui dérivent de ce framework pour consolider une vision cognitive à propos du changement linguistique.

\section{Abstract:}

In this article, we work with the category of reanalysis to shed light on a series of changes in the history of the Spanish language, and thereby to overcome the mere description level with the purpose of achieving the core goal of Science: theoretical explanation. In order to accomplish our mission, we base our work on the framework drawn up by Langacker (1977). The theoretical Langackerian framework sets a clear concept of reanalysis and produces a clear distinction of their types, causes, and effects. Firstly, we will make a concise presentation of Langacker's notions, above all, those regarding to the types of reanalysis. Then we will focus on some diachronic processes of the Spanish language that can be clarified in terms of the Langackerian category. Finally, we will expose some corollaries resulting from this framework to underpin a cognitive view of linguistic change.

\section{Palabras clave:}

Reánalisis; cambio lingüístico; diacronía castellana.

Mots clés:

Réanalyse ; changement linguistique ; diachronie castillane. 
Key words:

Reanalysis; linguistic change; Spanish diachrony.

Fecha de recepción: $\quad 31 / 05 / 2012$

Fecha de aceptación: $\quad$ 01/06/2012

\section{La categoría de reanálisis}

Cuando se constata un cambio lingüístico (ora fonético, ora sintáctico), tenemos la sensación de estar ante un eslabón perdido y, por ello, el espíritu científico anhela la causalidad, esto es, quiere llegar a la fuente de la explicación con sus categorías, sus hipótesis y sus leyes. La historia del español necesita formular constructos que permitan no solo describir, sino explicar los cambios ocurridos en su devenir, en su ritmo diacrónico. Por ejemplo, Vossler (1943) logró distinguir dos fenómenos diferentes que suelen confundirse en la mirada ignara sobre la historia del castellano: el sustantivo mente (como intelecto) no puede ser considerado como un reflejo del término mente del latín vulgar (cuyo significado era 'índole u opinión'). Se debe postular que el sustantivo en mención es un influjo tardío del latín culto y, en consecuencia, se debe categorizar como un préstamo. En cambio, el sufijo adverbializador -mente sí se puede comprender como una evolución a partir del término del latín vulgar (Fischer, 2007). Como arguye correctamente Vossler, la categoría de préstamo puede explicar mejor la introducción del sustantivo mente en el caudal léxico castellano, dado que suponer una vía patrimonial incurriría en la falencia de postular un cambio súbito contraintuitivo: «Va contra la ley de los cambios semánticos el que un significado relativamente estricto, como 'intelecto', proceda sin interrupción de otro relativamente amplio como 'índole'» (Vossler, 1943: 106. Son nuestras las cursivas).

Según Wright (1990), el vocablo latino messare 'arrancar' se fue especializando progresivamente hasta llegar a significar 'arrancar la barba o el cabello con las manos', como se puede pernotar en un texto de Lope de Vega (Fuenteovejuna): "Llévanle a una casa muerto/ y a porfía, quien más puede mesa su barba y cabello/ y apriesa su rostro hieren». En rigor, este uso se retrotrae hasta el Poema de Mío Cid. 
Hay muchos fenómenos en la diacronía castellana que evidencian procesos de esa índole: a partir del árabe miskîn 'pobre' se derivó el término castellano mezquino generándose un desarrollo peyorativo evidente y muy revelador sobre la categorización metafórica de la pobreza (Santos Domínguez \& Espinosa Elorza, 1996); en el siglo XVI se creó el término erudito con el significado de desbastado (i.e. 'que ya no es rudo'); se dio en castellano un fenómeno de decoloración [bleaching] semántica por el cual el verbo léxico babeo se destiñó hasta llegar a ser el simple auxiliar haber; finalmente, la palabra melancolía era la forma helénica de 'atrabilis' y su significado prístino estaba ligado al sentimiento colérico o atrabiliario; gradualmente, melancolía fue adquiriendo el sentido de tristeza profunda con el cual se asocia en nuestros días.

En la medida en que la ciencia no solo describe, sino explica, nuestro objetivo es utilizar una categoría epistémica que pueda brindarnos un marco de intelección para todos estos procesos. Creemos que la noción de reanálisis, tal como fue propuesta inicialmente por Langacker, es la categoría con la cual podemos explicar una parte importante de cambios ocurridos en nuestra lengua. De esa manera, haremos un avance teórico respecto de los estudios meramente descriptivos sobre la historia del castellano como lengua históricamente constituida.

El reanálisis es visto como uno de los principales mecanismos del cambio lingüístico y, en esencia, consiste en una modificación en algún ítem de la gramática que no se expresa superficialmente en la lengua, por lo menos de manera inmediata. Esto es, el cambio se produce en la configuración interna del sistema, pero no se ve reflejado en las emisiones idiomáticas patentes. Según Langacker, el reanálisis es el «change in the structure of an expression or class of expressions that does not involve any immediate or intrinsic modification of its surface manifestation» (1977: 58). Harris y Campbell (1995: 61) ofrecen una definición similar: «a mechanism which changes the underlying structure of a syntactic pattern and which does not involve any immediate or intrinsic modification of its surface manifestation». De esta manera, se puede establecer que se trata de una categoría asentada en la lingüística histórica y de gran utilidad para explicar los procesos históricos de la lengua castellana (Rodríguez 
Molina, 2011), aunque no es monolítica: el reanálisis se ha objetado en la medida que puede considerarse como un mero recurso ad hoc, esto es, carente de valor explicativo (Mc Daniels, 2003).

Los dos grandes tipos de reanálisis sintáctico son la resegmentación y la reformulación. Mientras que la resegmentación entraña una modificación en los límites morfémicos, la reformulación entraña un nivel de mayor abstracción: «is reanalysis that involves aspects of structure more abstract than the ocurrence and placement of morpheme boundaries» (Langacker, 1977: 79).

La resegmentación, a su vez, se puede dividir en a) pérdida de límite morfémico, b) creación de límite morfémico y c) desplazamiento de límite morfémico. En aras de la simplicidad, el desplazamiento de límite morfémico (boundary shift) se puede entender como una suma de las dos primeras, dado que "when $\mathrm{XY}-\mathrm{Z}$ is reanalyzed as $\mathrm{X}-\mathrm{YZ}$, we could simple say that the original morpheme boundary (between $\mathrm{Y}$ and $\mathrm{Z}$ ) is lost and that a new boundary (between $\mathrm{X}$ and $\mathrm{Y}$ ) is created» (Langacker, 1977: 66).

La reformulación puede ser de dos tipos: la puramente sintáctica y la que tiene efectos semánticos. Asimismo, la reformulación semántica puede operar en elementos particulares o puede aplicarse a la estructura global. Cuando opera en elementos particulares, tenemos tres casos: a) pérdida de una unidad semántica, b) adición de una unidad semántica y c) cambio en el valor semántico. La reformulación con efectos semánticos que incide en la estructura global se denomina reformulación construccional, dado que «it involves modification of the type of construction the sentence represents» (Langacker, 1977: 85).

\section{Aspectos de la diacronía castellana}

En esta parte del artículo, aplicaremos el framework langackeriano a ciertos casos de la historia de la lengua castellana. En primer lugar, presentaremos casos de resegmentación y, en segunda instancia, casos de reformulación (especialmente, reformulaciones con efectos semánticos). Los datos proceden de distintas fuentes (Lapesa, 1981; Cano Aguilar, 
https://doi.org/10.46744/apl.201201.002

2005; Penny, 2006; el CORDE, entre otros) y son datos muy bien consolidados en la tradición de la lingüística hispánica. Obviamente, la interpretación es personal y, en algunos casos, es algo conjetural (aunque, esperamos, formular hipótesis dotadas de plausibilidad).

\subsection{Casos de Resegmentación}

2.1.1 A partir de la expresión latina dum interim, se generó en castellano la expresión temporal do mientre. Como recuerdo de la expresión latina, se trataba de una secuencia bimorfemática $\left(d_{0}+\right.$ mientre $)$ y a partir de esta secuencia se dio el siguiente cambio:

\section{do mientre $>$ domientre}

Esto es, se dio una pérdida de límite morfémico. Este cambio generó una cadena diacrónica: domientre $>$ demientre $>$ mientras. El paso del latín al castellano implicó un boundary shift: dum - interim $>$ do - mientre (desplazamiento de $m$ del final del término dum al inicio del término interim). Evidentemente, el morfema do fue perdiendo claridad significativa en un proceso conocido como bleaching [decoloración]. Ello implicó la pérdida del límite morfémico y la consecuente pérdida de todo el segmento, lo que nos lleva al resultado final: mientras.

2.1.2 En castellano, se generó una forma: vos + otros. En esa forma histórica hubo una pérdida de límite morfémico y la forma resultante es vosotros. Este resultado ha quedado vigente en el castellano ibérico. La fusión (consecuencia de la pérdida del límite morfémico) se puede refrendar con las intuiciones de los hablantes que captan la referida forma como un solo pronombre. Sin embargo, en un tiempo de la historia castellana, los hablantes eran conscientes de la estructura bimorfemática $(v o s+o t r o s)$ y ello explicaría el origen de la forma analógica: nos + otros. Asimismo, la forma analógica también sufrió la pérdida del límite morfémico y devino en la actual nosotros.

2.1.3 A partir de la forma latina hipotética *verruculu, se generó la forma del castellano medieval verrojo en virtud de una serie de procesos 
fonéticos explicados por los gramáticos históricos. Ahora bien, luego se da el cambio berrojo > cerrojo explicable por etimología popular. Este cambio entraña la creación de un límite morfémico (boundary creation), dado que los hablantes interpretaron que cerrojo era un derivado del verbo cerrar. En consecuencia, tenemos lo siguiente:

$$
\begin{aligned}
& \text { berrojo }>\text { cerrojo } \\
& \text { cerrojo }>\text { cerr }+ \text { ojo }
\end{aligned}
$$

El cambio (1) se explica por etimología popular, una interpretación errónea del hablante que conduce a una nueva forma que es santificada por el uso. El cambio (2) es una interpretación de los hablantes que obedece a un reanálisis en el modo de la creación de un límite morfémico. Este reanálisis ha tenido como efecto una especialización semántica (la extensión de berrojo era mayor que nuestro actual cerrojo).

\subsection{Casos de Reformulación}

2.2.1 En latín, la palabra pinus era gramaticalmente femenina. En castellano, dio pino que se reanalizó como sustantivo masculino. Este reanálisis implica un cambio semántico (de femenino a masculino): $p i n-o$, en el que $-o$ se reanaliza como masculino (esto es, pierde su prístina interpretación como femenino). En los albores del castellano, había palabras femeninas terminadas en -o y algunas han quedado como tales (nao, mano, etc.). Sin embargo, algunas palabras como pino u olmo sufrieron el referido reanálisis. Evidentemente, este caso de reanálisis tiene como objetivo hacer más patente la generalización que opera en la mente del hispanohablante (-o es una marca de masculinidad).

2.2.2 En latín, la palabra opera era una forma con significado plural y pertenecía a los sustantivos neutros. Como tal desembocó en la palabra castellana obra que fue reanalizada como singular y femenina. Este shift semántico se explica en virtud de que había mucha vacilación tras la pérdida de la categoría neutro para los sustantivos. 
2.2.3 A partir de la forma latina cantaveram, en castellano se desarrolló la forma cantara ( $<$ cantaram). El valor de la prístina forma castellana era pluscuamperfecto del indicativo (sirve para designar un hecho pasado dentro del pasado). Este valor perduró hasta la época áurea del castellano conocida como Siglo de Oro. Luego, hubo un cambio semántico: cantara pasó a designar el subjuntivo. El reanálisis se puede bosquejar de la siguiente manera:

\begin{tabular}{|c|c|}
\hline INDICATIVO & SUBJUNTIVO \\
\hline cantara & cantara \\
\hline
\end{tabular}

Una de las pruebas más nítidas de la gradualidad del cambio es que, en nuestro cronolecto, haya expresiones en las cuales todavía perviva el valor del pluscuamperfecto de indicativo. Por ejemplo, en un enunciado como «el poemario que Vallejo escribiera en su juventud», el verbo no se puede interpretar como subjuntivo (en efecto, equivale a 'había escrito', pluscuamperfecto de indicativo).

2.2.4 Otro shift semántico se puede notar en la palabra cadera (sinónimo actual de anca). La forma clásica cathedra se expresaba en latín vulgar como cathegra y, de allí, pasó al castellano como cadera (con el significado de 'nalga'). Como efecto de una metonimia, se dio el cambio semántico al sentido que tiene hasta la actualidad ('parte lateral y saliente de la región anatómica de la pelvis').

2.2.5 Los cambios semánticos abundan en la historia del castellano, lo que confirma una intuición de Langacker (1977): el shift semántico es el tipo de reformulación más frecuente. Este tipo de reanálisis ha ocurrido con dinero 'valor económico en general' ( $<$ denariu 'moneda específica'), asir 'tomar o coger en general' ( < assir 'coger por el asa'), casa 'vivienda en general' $(<$ casa 'cabaña'). El cambio semántico puede implicar la pérdida del valor semántico inicial, lo que ha ocurrido, por ejemplo, con la palabra siniestro $(<$ sinister 'izquierdo'). Actualmente, siniestro designa 'avieso' o 'daño terrible' (por ello, una manera hiperonímica de hablar de un incendio es decir 'el siniestro...'). El shift semántico puede tener un efecto ameliorativo, lo que se observa en el cambio ministro 'servidor' ( $<$ ministru) a ministro 'funcionario importante'. 
Mas, también, puede tener un efecto peyorativo el sufijo -ote, con valor inicial de aumentativo, ha desarrollado un valor peyorativo cuando se aplica a ciertos sustantivos. Como ha sido explicado por Langacker, el shift semántico involucra, generalmente, dos movimientos en el lenguaje: adición y pérdida de rasgos semánticos. Por ejemplo, la palabra castellana lunes procede de la frase latina dies Lunae (los días dedicados a la Luna). En nuestro actual vocablo lunes, se observa, por lo tanto, la supresión de un rasgo semántico (el valor significativo referido a la Luna) y la adición de un rasgo semántico (el valor significativo de primer día de la semana). Asimismo, los vaivenes históricos pueden erosionar un rasgo semántico, dado que las palabras o expresiones de nuestro lenguaje están ligadas indisolublemente a nuestras formas de vida. Esto ha ocurrido recientemente con la lexía luna de miel cuyo significado originario era el 'primer mes en la vida íntima conyugal'. La vorágine de nuestro tiempo hace muy difícil que dure tanto una luna de miel, razón por la cual se interpreta austeramente como 'tiempo de intimidad conyugal inmediatamente posterior al matrimonio', con lo cual se produce un reanálisis como reformulación del rasgo semántico concerniente a la duración temporal.

2.2.6 En el latín había un tiempo compuesto cantare habebam, que dio paso a la expresión perifrástica castellana cantar (b)ía. La estructura latina se sustentaba en la siguiente configuración: infinitivo más imperfecto. Tal estructura pasó al castellano arcaico con el mismo valor y, por ello, se respetó la estructura perifrástica. La naturaleza compuesta de la expresión se prueba por el hecho de que se podía intercalar un clítico en su estructura. El cambio sintáctico implica la formación del condicional (como voz simple):

$$
\text { cantar (b)ía > cantaría }
$$

La reformulación construccional que origina el condicional castellano se explica por el hecho semántico de que el condicional se puede entender como un futuro dentro del pasado.

2.2.7 En la historia del castellano, hay una frecuente aplicación de la reformulación reconstruccional, según la cual una forma perifrástica 
se reduce a una palabra (como suma de los significados de los elementos de la perífrasis o como la creación de un nuevo valor semántico). Así, tenemos varios casos como los siguientes:

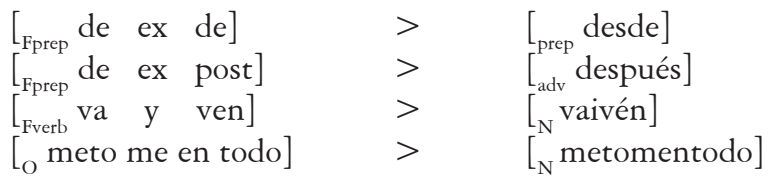

\section{La naturaleza del cambio lingüístico}

Tal como se ha definido y aplicado, la noción de reanálisis se puede entender como una vía o una condición para la gramaticalización, lo que está sugerido por el mismo Langacker cuando señala que «a reanalysis may have the effect of creating conditions that trigger another reanalysis» (1977: 95). En tal sentido, ofrece una perspectiva fértil en la lingüística histórica. La noción de gramaticalización fue usada por Meillet (1912: 131) para dar cuenta de «l'attribution du charactère gramatical à un mot jadis autonome» $y$ ha sido definida como «the increase of the range of a morpheme advancing from a lexical to a gramatical or from a less grammatical to a more grammatical status» (Kurylowicz, 1965: 69). En la diacronía de las lenguas, la gramaticalización es un proceso importante que, además, revela algo señalado por el nuevo enfoque cognitivo: la relación entre gramática y léxico es un continuum. La gramaticalización es una noción clave para entender la dinámica del cambio sintáctico en las lenguas (Roberts \& Roussou, 2003).

En castellano, la gramaticalización ha ocurrido en el siguiente proceso res nata 'cosa nacida' $>$ nada (así como en nati 'los nacidos' $>$ nadie). Inicialmente, nada tenía un valor léxico positivo y, gradualmente, ha desarrollado un valor de término de polaridad negativa para fijar el parámetro de la concordancia negativa que se observa en el castellano. En tal sentido, se ha despojado de su valor léxico y ha desarrollado un valor gramatical. Dado que la gramaticalización parece la tendencia más vital del cambio lingüístico, se suele soslayar la presentación del fenómeno complementario: la desgramaticalización (Norde, 2009), esto 
es, la atribución de un valor léxico a un término que tenía un estatus puramente gramatical. En castellano, el proceso ha ocurrido en formas como pro (pierde su estatus de preposición y adquiere un valor nominal en enunciados como «Debes evaluar los pros y los contras antes de tomar una decisión») o super- (pierde su valor de elemento compositivo $\mathrm{y}$ adquiere un valor adjetival en algunos enunciados coloquiales como «Tu padre es un tipo súper»). Obviamente, también hay reanálisis en la desgramaticalización.

Para dar cuenta del mecanismo del reanálisis, Langacker se refiere a las causas como factores que contribuyen al desarrollo del cambio o lo facilitan. En este sentido, Langacker menciona algunos factores (verbigracia, la tendencia a la simplicidad de la señal), pero la referencia causal más fuerte es la propensión a la transparencia. Como señala Langacker: «Language change reflects the pressure to achieve linguistic optimality, but linguistic optimality has numerous dimensions reflecting the multi-faceted character of language, and the tendencies to achieve these different kinds of optimality are often in opposition to one another» (1977: 128). El reanálisis está, pues, involucrado en la propensión a la simetría que sería la ley fundamental del cambio lingüístico.

Una cuestión intrincada, pero esencial, es ¿por qué las lenguas manifiestan esa propensión a la simetría? Un sistema simétrico es óptimo en la medida en que expresa con transparencia el engarce (link) entre los dos polos del lenguaje: el polo fonológico (patente) y el polo semántico (subyacente). La mente humana necesita salir de la opacidad para cimentar mejor su manera de insertarse en el mundo. Ahora bien, dado que hay varios modos de aplicar la optimidad (es decir, varias maneras de concretar la simetría lingüística), se genera un conjunto de tensiones que pueden desembocar en algunas encrucijadas verbales. La presión por salir de estas encrucijadas idiomáticas explicaría, en gran parte, la necesidad de los cambios lingüísticos. Los diversos tipos de reanálisis, las diferentes causas del mecanismo y los variados efectos implicados configuran el escenario de la lingüística histórica: una ciencia que estudia sistemas que expresan una simetría dinámica. 


\section{BIBLIOGRAFÍA}

CANO AGUILAR, Rafael. El español a través de los tiempos. Madrid: Arco Libros, 2005.

FISCHER, Olga. Morphosyntactic Change. Functional and Formal Perspectives. Oxford: Oxford University Press, 2007.

MC DANIELS, Todd. «What's wrong with reanalysis». Toronto Working Papers in Linguistics, 21, pp. 81-88; 2003.

HARRIS, Alice C. and Lyle CAMPBELL. Historical Syntax in Crosslinguistic Perspective. Cambridge: Cambridge University Press, 1995.

KURYLOWICZ, J. «The Evolution of Grammatical Categories». Esquisses Linguistiques II. Munich: Verlag; pp. 38-54; 1965.

LANGACKER, Ronald W. «Syntactic Reanalysis». Charles N. Li (ed.) Mechanisms of syntactic change. Austin: University of Texas Press; pp. 57-139; 1977.

LAPESA, Rafael. Historia de la lengua española. Madrid: Gredos, 1981.

MEILLET, A. «L'evolution des formes grammaticales». Scientia, 7, pp. 6-24; 1912.

NORDE, Muriel. Degrammaticalization. Oxford: Oxford University Press, 2009.

PENNY, Ralph. Gramática histórica del español. Barcelona: Editorial Ariel, 2006.

ROBERTS, Ian and Anna ROUSSOU. Syntactic Change. A Minimalist Approach to Grammaticalization. Cambridge: Cambridge University Press, 2003. 
RODRÍGUEZ MOLINA, Javier. La gramaticalización de los tiempos compuestos en español antiguo: cinco cambios diacrónicos. Tesis doctoral. Madrid: Universidad Autónoma de Madrid, 2011.

SANTOS DOMÍNGUEZ, Luis y Rosa María ESPINOSA ELORZA. Manual de Semántica Histórica. Madrid: Editorial Síntesis, 1996.

VOSSLER, Karl. Filosofía del lenguaje. Buenos Aires: Losada, 1943.

WRIGHT, Roger. «Semantic Change in Romance Words for 'CUT'». Henning Andersen \& Konrad Koerner (eds.) Historical Linguistics 1987. Amsterdam/Filadelfia: John Bemjamins, pp. 553-561; 1990.

\section{Correspondencia:}

\section{Raymundo Casas Navarro}

Docente del Departamento Académico de Lingüística de la Facultad de Letras y Ciencias Humanas de la Universidad Nacional Mayor de San Marcos.

Correo electrónico: casasnavarro1@hotmail.com 